Article

\title{
Study on the Association among Mycotoxins and other Variables in Children with Autism
}

\author{
Barbara De Santis ${ }^{1, *}$, Maria Elisabetta Raggi ${ }^{2}$, Giorgio Moretti ${ }^{1}$, Francesco Facchiano ${ }^{3}$, \\ Alessandra Mezzelani ${ }^{4}$, Laura Villa ${ }^{2}$, Arianna Bonfanti ${ }^{2}$, Alessandra Campioni ${ }^{1}$, \\ Stefania Rossi ${ }^{3}$, Serena Camposeo ${ }^{5}$, Sabina Soricelli ${ }^{1}$, Gabriele Moracci ${ }^{1}$, \\ Francesca Debegnach ${ }^{1}$, Emanuela Gregori ${ }^{1}$, Francesca Ciceri ${ }^{2}$, Luciano Milanesi ${ }^{4}$, \\ Anna Marabotti ${ }^{2,6, *}$ and Carlo Brera ${ }^{1}$
}

1 GMO and Mycotoxin Unit, Department of Veterinary Public Health and Food Safety, Italian National Institute for Health, Viale Regina Elena, 299-00161 Roma, Italy; giorgio.moretti87@gmail.com (G.M.); alessandra.campioni@hotmail.it (A.C.); sabina.soricelli@gmail.com (S.S.); gabriele.moracci@iss.it (G.M.); francesca.debegnach@iss.it (F.D.); emanuela.gregori@iss.it (E.G.); carlo.brera@iss.it (C.B.)

2 Scientific Institute, IRCCS Eugenio Medea, Bosisio Parini, Via Don Luigi Monza, 20-23842 Bosisio Parini, Lecco, Italy; mariaelisabetta.raggi@bp.lnf.it (M.E.R.); laura.villa@bp.lnf.it (L.V.); arianna.bonfanti@bp.lnf.it (A.B.); francescaciceri@libero.it (F.C.)

3 Department of Oncology and Molecular Medicine, Italian National Institute for Health, viale Regina Elena, 299-00161 Roma, Italy; francesco.facchiano@iss.it (F.F.); stefania.rossi.2103@gmail.com (S.R.)

4 National Council of Research, Institute of Biomedical Technologies, Via F.lli Cervi 93, 20090 Segrate, Milano, Italy; alessandra.mezzelani@itb.cnr.it (A.M.); luciano.milanesi@itb.cnr.it (L.M.)

5 Scientific Institute, IRCSS Eugenio Medea, 72100 Brindisi, Italy; serena.camposeo@libero.it

6 Department of Chemistry and Biology “A. Zambelli”, University of Salerno, Via Giovanni Paolo II, 132, 84084 Fisciano, Salerno, Italy

* Correspondence: barbara.desantis@iss.it (B.D.S.); anna.marabotti@gmail.com (A.M.); Tel.: +39-06-4990-2367 (B.D.S.); +39-089-969-583 (A.M.)

Academic Editor: James J. Pestka

Received: 17 February 2017; Accepted: 23 June 2017; Published: 29 June 2017

\begin{abstract}
Environmental factors and genetic susceptibility are implicated in the increased risk of autism spectrum disorder (ASD). Mycotoxins are agricultural contaminants of fungal origin that represent real risk factors for human health and especially for children. Thus, the main hypothesis of this work is that the deterioration of the clinical manifestation of autism in children may result from the exposure to mycotoxins through the consumption of contaminated food. Within a cross-sectional study, a group of autistic children $(n=172)$ and a group of controls $(n=61)$ (siblings and non-parental) were recruited in North and South Italy. All children had blood and urine samples taken, for testing some mycotoxins by a LC-MS/MS validated method. Blood samples were also tested for assessing specific IgG against food and fungal antigens and cytokines. The analyses outputs highlighted statistically significant differences comparing mycotoxins levels between (i) children groups both in urine (deoxynivalenol and de-epoxydeoxynivalenol, $p=0.0141$ and $p=0.0259$, respectively) and serum (aflatoxin M1, ochratoxin A and fumonisin B1, $p=0.0072, p=0.0141$ and $p=0.0061$, respectively); (ii) a group of selected fungal IgGs, and IgGs against wheat and gluten and (iii) cytokines. These results suggest the need for a deeper examination of the role that mycotoxins may have on the etiology of ASD.
\end{abstract}

Keywords: autism syndrome; mycotoxins; environment; exposure; IgG; cytokine/chemokine 


\section{Introduction}

Mycotoxins are agricultural contaminants of fungal origin that may occur in almost all types of crops involving the whole agri-food chain. Indeed, because of their extreme heat stability, mycotoxins are present in untreated seeds up to processed foods and feed as well.

Mycotoxin-producing molds are able to grow at any latitude worldwide and, in the last few years, have been creating a real burden in agriculture that was estimated by the Food and Agriculture Organization (FAO) as $25 \%$ of the world's crops being affected each year. The main consequences for the agri-food chain and human and animal health entail direct implications from an economical point of view, with worldwide annual losses of around 1 billion metric tons of foodstuff and food products and for health-related issues such as the incidence of various diseases, like cancer, gastrointestinal (GI) and neurological impairment [1].

Mycotoxins occur in plant and animal origin products under modified forms or as a cocktail of compounds possibly increasing the effect of their toxicity due to synergistic and/or additive effects.

Due to their chemical structure, mycotoxins have different target organs with a wide range of effects, such as intestinal permeability, oxidative stress, inflammation and fibrosis in some target organs leading to nephrotoxicity, hepatotoxicity, neurotoxicity and immunotoxicity $[2,3]$.

Epidemiological evidences are reported in literature where aflatoxins ( $\mathrm{AFs}$ ) have been extensively associated with liver cancer, hepatitis B, morbidity in children suffering from kwashiorkor (a severe protein-energy malnutrition syndrome), stunting and even death in extremely acute exposures [4]. Other mycotoxins were associated to various outbreaks as well: deoxynivalenol (DON) strongly correlates to GI disturbs and fumonisins (FBs) are related to esophageal cancer and neural tube defects. Zearalenone (ZEA) results to be involved in thelarche and gliotoxin (GLIO) is possibly implicated in the etiology of immunologic deficiency. Ochratoxin A (OTA), classified by the International Agency for Research on Cancer (IARC) in Group 2b, i.e., possibly carcinogenic for humans, is suspected to be weakly mutagenic, maybe through oxidative DNA damage, able to exert a causal effect on nephropathies (Balkan Endemic Nephropathy) as well as to increase the incidence of transitional cell (urothelial) urinary cancers [5]. Lastly, it has also been reviewed that AFs, DON and FBs are all involved in intestinal damage through inhibition of protein synthesis (AFs and DON), an increase in systemic proinflammatory cytokines (DON) and inhibition of ceramide synthase (FBs) [6]. The development and the use of biomonitoring helped in food safety to explore exposure scenarios over more traditional approaches. Up to now, as for mycotoxin concern, a number of studies have already been published reporting the scientific evidence of the validation of aflatoxin M1 (AFM1), OTA, DON and FBs as biomarkers of exposure [2,7-11].

The term "autism spectrum disorder" (ASD) is generally referred to a group of neurodevelopmental disorders characterized by a broader phenotype, including typical features such as stereotyped or repetitive behaviours, impaired social activities, and restricted verbal and non-verbal communication [12]. The symptoms manifest generally by 3 years of age, and there is the evidence that often ASD children experience a loss of skills between 18 and 21 months of age, on average, after a relatively normal development [13]. Autism affects more males than females: recent epidemiological studies suggest that the ratio in the prevalence/incidence of ASD is in the range of 2-5:1 male:female [14].

The median of prevalence estimates of ASD was reported to be $62 / 10,000$ in a systematic review of epidemiological surveys of autistic disorder worldwide. The prevalence is continuously increasing over time, and this phenomenon can be partially related to the improvement of the diagnostic criteria, switching from other developmental disabilities to ASD [15]. Genetic factors are considered to play an important role in ASD etiology and over 700 genetic loci are implicated in ASD to date. However, most of these are either rare variants, or are common variants in the general population conferring only a small risk for ASD. Additionally, the genomic architecture of ASD is heterogeneous and complex, and it is very difficult to infer common molecular mechanisms. For all these reasons, it has long been hypothesized that gene-environment interaction could be involved in ASD pathogenesis. Among 
environmental agents, air pollutants, heavy metals, organic toxicants, pesticides, phthalates have long been investigated, as well as seasonal, social and familial environmental factors [16].

Often, ASD is associated to comorbid situation such as cognitive delays, epilepsy, oxidative stress, GI disorders and inflammation. Recently, more attention has been paid on nutritional factors, considering the increasingly important role of gut microbiota and of the so-called "microbiota-gut-brain axis" in determining the human health and the correct development of the central nervous system (CNS) [17]. Gut microbiota, indeed, regulates metabolism and homeostasis and plays a key role in controlling the CNS functions through neural, endocrine and immune pathways [18]. Thus, gut dysbiosis (microbial imbalance) not only leads to inflammatory bowel diseases but also to systemic inflammation altering the CNS functions as described in some neuropsychiatric conditions, including ASD. Some studies have already been conducted to define the bacterial gut composition of ASD patients although with discordant results. Very recently, Strati and collaborators [19] analyzed, for the first time, both the gut microbioma and mycobioma (fungal profile) of ASD children and healthy controls. Interestingly, they found alterations in both the bacterial and fungal kingdom. Specifically, as for prokaryotes, they confirm that ASD subjects displayed a reduction of Bacteroidetes, Dialister and Veillonella as well as an increase of Lactobacillus. Regarding the fungal composition, a relative abundance (more than double) of Candida was detected in ASD patients versus controls revealing that also fungi participate in ASD dysbiosis and providing a new therapeutic target.

Moreover, the observation that often in ASD patients there are recurrent GI abnormalities with a correlation with the severity of autistic symptoms [20] has led to the development of some diets, such as the gluten-free/casein-free diet to reduce or abolish these problems. Interestingly, several studies pointed out that this diet could induce positive cognitive and behavioral effects on ASD children with GI disorders [21], although further data are needed to fully understand the possible mechanisms of action for this diet.

The overlap of the toxicological effects of some mycotoxins with several autistic features e.g., oxidative stress, leaky gut and inflammation that, in turn, impair the microbiota-gut-brain axis, is remarkable. In this scenario, here the presence of Aflatoxin B1 (AFB1), aflatoxin M1 (AFM1), DON, de-epoxy deoxynivalenol-1 (DOM1), fumonisin B1 (FB1), GLIO, OTA and ZEA were has been quantitatively analysed by Ultra-Performance Liquid Chromatography [UPLC ${ }^{\circledR}$-MS/MS in urine and serum samples collected in a sizable set of ASD patients and controls. The levels found have been associated with measured variables such as a group of IgGs against fungal and food antigens and a group of cytokines/chemokines of pro-inflammatory signals and in immunity responses. This led us to speculate that mycotoxins exposure could have detrimental consequences and participate in the aggravation of medical comorbidities in autistic children.

\section{Results}

\subsection{Sample Selection}

The study enrolled a grand total of 233 children, split into autistic group $(n=172)$, a group of sisters or brothers of the autistics (siblings, $n=36$ ) and a group of non-parental children (non-parental group, $n=25$ ). In Table 1, the depiction of the group in terms of gender and age is detailed: 131170 (81.772.9\%) males; 2963 (18.327.1\%) females; age ranging between 2 and 12 years. As expected, boys were overrepresented in the ASD case group with a male/female ratio of 4.5/1. 
Table 1. Demographic and clinical characteristics of the subjects.

\begin{tabular}{cccc}
\hline & Autistics & Siblings & Non-Parental \\
\hline N of subjects (233 in total) & 172 & 36 & 25 \\
Sex (Male) & $81.9 \%$ & $41.6 \%$ & $56 \%$ \\
Male/Female ratio & $141 / 31$ & $15 / 21$ & $14 / 11$ \\
Age (mean \pm SD $)$ & $5.68 \pm 2.58$ & $7.95 \pm 3.84$ & $8.53 \pm 2.03$ \\
New-born length (cm) & $49.16 \pm 5.44$ & $50.20 \pm 2,44$ & $50.02 \pm 1,87$ \\
New-born weight (gr) & $3181.72 \pm 705.81$ & $3293.09 \pm 450.04$ & $3297.73 \pm 473.75$ \\
Head circumference (cm) & $34.55 \pm 2.83$ & $34.34 \pm 1.87$ & $34.08 \pm 1.40$ \\
Regressive pattern & $30.1 \%$ & - & - \\
Location (referred to Nord) & $72.9 \%$ & $66.7 \%$ & $87.05 \%$ \\
Non-verbal & $58(33.7 \%)$ & - & - \\
\hline Gastrointestinal problems & $26.6 \%$ & $2.6 \%$ & $2.1 \%$ \\
(referred to $N=233)$ & $*$ Standard deviation. & &
\end{tabular}

A questionnaire was submitted and responded by the parents or tutors of each child, in order to collect information about clinical issues in the past, with a particular focus on GI alterations and dietary habits. In Table 2 , the statistical analyses that gave significant $p$ values $(\leq 0.050)$ are reported.

Table 2. Results of the gastrointestinal problems between ASD group and control group (siblings and non-parental, summed).

\begin{tabular}{ccccc}
\hline Gastrointestinal Problems & Autistics & Control (All) & Odds Ratio & $\boldsymbol{p}$-Value \\
\hline Vomit & 65 & 18 & 4.28 & $\mathbf{0 . 0 2 4}$ \\
Diarrhoea & 83 & 26 & 2.41 & $\mathbf{0 . 0 5 0}$ \\
Constipated & 85 & 24 & 1.92 & 0.169 \\
Stomach-aches & 48 & 16 & 1.74 & 0.620 \\
Painful bowel & 60 & 23 & 0.65 & 0.389 \\
Gastroenteritis & 58 & 25 & 0.96 & 0.940 \\
Aerophagia & 60 & 16 & 8.07 & $\mathbf{0 . 0 2 4}$ \\
Abdominal bloating & 56 & 21 & 1.74 & 0.313 \\
Lack of appetite & 56 & 19 & 3.02 & 0.068 \\
Intestinal infections & 171 & 60 & 1.82 & 0.079 \\
Intestinal anomalies & 169 & 60 & 2.81 & $\mathbf{0 . 0 0 7}$ \\
\hline
\end{tabular}

From the list in Table 2, it is shown that children with ASD are approximately two to eight times more likely to report frequent vomit, diarrhea, abdominal bloating, aerophagia and intestinal anomalies $(p \leq 0.050$, in bold in Table 2$)$ than the control group.

\subsection{Mycotoxins Analysis}

Aflatoxin B1, M1, DON, DOM1, FB1, GLIO, OTA and ZEA were analysed by UPLC-MS/MS in urine and serum samples collected from the subjects. For this purpose, a method was developed and validated following international standards [22] and assessing the apparent recovery $\left(\mathrm{R}_{\mathrm{A}}\right)$, the signal suppression/enhancement (SSE) and the recovery for extraction $\left(\mathrm{R}_{\mathrm{E}}\right)$ [23].

\subsubsection{Method Validation}

In Table 3, a summary of the method performances obtained during the validation phase is reported. For both biological matrices (urine and serum), the limit of quantification (LoQ), the relative standard deviation (RSDr) and $\mathrm{R}_{\mathrm{A}}$ of the method, the SSE and the $\mathrm{R}_{\mathrm{E}}$ are shown. 
Table 3. Method performances in urine and serum.

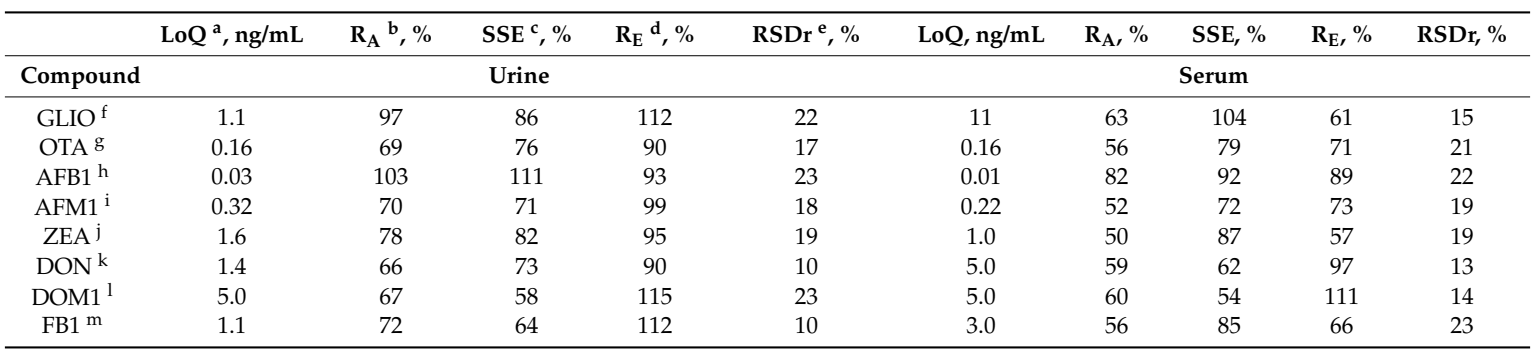

${ }^{a}$ Limit of Quantification; ${ }^{\mathrm{b}}$ Apparent recovery; ${ }^{\mathrm{c}}$ Signal Suppression/Enhancement; ${ }^{\mathrm{d}}$ Recovery for extraction;

e Relative Standard Deviation (repeatability); ${ }^{\mathrm{f}}$ Gliotoxin; ${ }^{\mathrm{g}}$ Ochratoxin A; ${ }^{\mathrm{h}}$ Aflatoxin B1; ${ }^{\mathrm{i}}$ Aflatoxin M1;

${ }^{\mathrm{j}}$ Zearalenone; ${ }^{\mathrm{k}}$ Deoxynivalenol; ${ }^{\mathrm{l}}$ De-epoxy deoxynivalenol-1; ${ }^{\mathrm{m}}$ Fumonisin B1.

Apparent recoveries values and matrix effects on signal (measured by SSEs) strongly varied depending on the analyte/matrix combination. The trend of SSE was comparable in both matrices. The strongest suppression was registered by DOM1 both in urine and serum. However, except for GLIO in serum and AFB1 in urine, all the other toxins gave suppression: important in the case of DON and DOM1, modest in all the other cases. As for apparent recoveries, urine gave general best performances in comparison with serum. The percentage values of RSDr, referred to the repeatability of spiked samples, ranged from 10 to $23 \%$.

\subsubsection{Occurrence}

In Tables 4 and 5, the description of the dataset (number of observations, percentage values, average, standard deviation, range (minimum-maximum values registered) and median) both as a whole and for each single group (autistic, siblings and non-parental) for the analysed mycotoxins, is reported.

Urine. Excepting for DOM1 and FB1, the percentages of samples with values above LoQ were more than $50 \%$ with a peak of $76 \%$ for OTA. Excepting for GLIO in all groups and DOM1 in non-parental, all mean values were below the LoQ. Scrutinizing the co-occurrence of the toxins in urine in the whole dataset, $3 \%$ of the samples $(n=5)$ showed positive values $(\geq$ LoQ) for the eight mycotoxins, while $32 \%(n=52)$ showed the presence of AFB1, AFM1 and OTA, and $9 \%(n=14)$ the presence of DON, FB1 and ZEA. DOM1 showed the lowest number of positive results $(28.4 \%$ in the whole group, $34.6 \%$ in the autistic group, $8 \%$ in siblings and $22.7 \%$ in the non-parental group).

Serum. Excepting for OTA levels in all groups and AFM1 in whole and autistic group, the percentages of positive samples were below $50 \%$, the highest percentage being for OTA in autistic children (85\%). Apart from OTA in all groups, all mean values were below the LoQ. Scrutinizing the co-occurrence of the toxins in urine in the whole dataset, no sample was positive for the eight mycotoxins, while $4 \%(n=9)$ showed the co-presence of AFB1, AFM1 and OTA and $2 \%(n=5)$ the presence of GLIO, AFB1, AFM1 and OTA. ZEA showed the lowest number of positive results $(5.4 \%$ in the whole group, $5.2 \%$ in the autistic group, $8.2 \%$ in siblings and 0 in the non-parental group).

Table 4. Description of the whole dataset for the mycotoxins analysed in urine samples.

\begin{tabular}{|c|c|c|c|c|c|c|}
\hline Group & Observations & $\%$ Positive & Mean (ng/mL) & $\mathrm{SD}(\mathrm{ng} / \mathrm{mL})$ & $\operatorname{Min}-\operatorname{Max}(n g / m L)$ & Median (ng/mL) \\
\hline Whole Group & $163 / 233 *$ & & & & & \\
\hline GLIO & & $71.6 \%$ & 8.2 & 1.5 & $0-114.7$ & 3.9 \\
\hline OTA & & $74.8 \%$ & 0.09 & 0.09 & $0-0.54$ & 0.08 \\
\hline AFB1 & & $66.2 \%$ & 0.01 & 0.01 & $0-0.06$ & 0.02 \\
\hline AFM1 & & $62.6 \%$ & 0.12 & 0.12 & $0-0.70$ & 0.15 \\
\hline ZEA & & $51.0 \%$ & 0.7 & 1.1 & $0-6.5$ & 0.8 \\
\hline $\mathrm{DON}$ & & $38.6 \%$ & 0.7 & 1.1 & $0-4.6$ & 0.0 \\
\hline DOM1 & & $27.0 \%$ & 2.1 & 4.2 & $0-19.5$ & 0.0 \\
\hline FB1 & & $44.5 \%$ & 0.4 & 1.1 & $0-12.4$ & 0.0 \\
\hline
\end{tabular}


Table 4. Cont.

\begin{tabular}{|c|c|c|c|c|c|c|}
\hline Group & Observations & $\%$ Positive & Mean (ng/mL) & $\mathrm{SD}(\mathrm{ng} / \mathrm{mL})$ & $\operatorname{Min}-\operatorname{Max}(\mathrm{ng} / \mathrm{mL})$ & Median (ng/mL) \\
\hline Autistic Group & 114 & & & & & \\
\hline GLIO & & $69.2 \%$ & 7.6 & 13.6 & $0-82.7$ & 3.5 \\
\hline OTA & & $70.2 \%$ & 0.09 & 0.10 & $0-0.54$ & 0.08 \\
\hline AFB1 & & $63.2 \%$ & 0.01 & 0.01 & $0-0.06$ & 0.02 \\
\hline AFM1 & & $60.7 \%$ & 0.12 & 0.13 & $0-0.70$ & 0.15 \\
\hline ZEA & & $50.5 \%$ & 0.8 & 1.2 & $0-6.5$ & 0.8 \\
\hline $\mathrm{DON}$ & & $46.5 \%$ & 0.9 & 1.2 & $0-4.6$ & 0.0 \\
\hline DOM1 & & $32.4 \%$ & 2.5 & 4.5 & $0-19.5$ & 0.0 \\
\hline FB1 & & $43.9 \%$ & 0.5 & 1.3 & $0-12.4$ & 0.0 \\
\hline Siblings & 25 & & & & & \\
\hline GLIO & & $80.0 \%$ & 11.6 & 24.7 & $0-114.7$ & 5.3 \\
\hline OTA & & $84.0 \%$ & 0.09 & 0.07 & $0-0.27$ & 0.08 \\
\hline AFB1 & & $80.0 \%$ & 0.01 & 0.01 & $0-0.03$ & 0.02 \\
\hline AFM1 & & $84.0 \%$ & 0.16 & 0.11 & $0-0.43$ & 0.15 \\
\hline ZEA & & $48.0 \%$ & 0.5 & 0.7 & $0-2.8$ & 0.0 \\
\hline $\mathrm{DON}$ & & $16.0 \%$ & 0.4 & 1.0 & $0-3.7$ & 0.0 \\
\hline DOM1 & & $8.0 \%$ & 0.8 & 2.8 & $0-12.0$ & 0.0 \\
\hline FB1 & & $56.0 \%$ & 0.4 & 0.5 & $0-2.0$ & 0.5 \\
\hline Non-Parental & 24 & & & & & \\
\hline GLIO & & $77.3 \%$ & 7.7 & 9.8 & $0-39.7$ & 4.2 \\
\hline OTA & & $87.5 \%$ & 0.09 & 0.05 & $0-0.19$ & 0.08 \\
\hline AFB1 & & $66.7 \%$ & 0.01 & 0.01 & $0-0.02$ & 0.02 \\
\hline AFM1 & & $54.2 \%$ & 0.08 & 0.09 & $0-0.25$ & 0.15 \\
\hline ZEA & & $59.1 \%$ & 0.6 & 0.8 & $0-3.17$ & 0.78 \\
\hline $\mathrm{DON}$ & & $25.0 \%$ & 0.4 & 0.8 & $0-2.3$ & 0.0 \\
\hline DOM1 & & $22.7 \%$ & 1.8 & 3.6 & $0-11.4$ & 0.0 \\
\hline FB1 & & $36.4 \%$ & 0.2 & 0.4 & $0-1.5$ & 0.0 \\
\hline
\end{tabular}

* Due to the low amount of samples collected from some children, it was not always possible to carry out all the analyses planned for the study.

Table 5. Description of the whole dataset for the mycotoxins analysed in serum samples.

\begin{tabular}{|c|c|c|c|c|c|c|}
\hline Group & Observations & $\%$ Positive & Mean (ng/mL) & Std Dev (ng/mL) & $\operatorname{Min}-\operatorname{Max}(\mathrm{ng} / \mathrm{mL})$ & Median (ng/mL) \\
\hline Whole Group & $213 / 233 *$ & & & & & \\
\hline GLIO & & $21.2 \%$ & 2.3 & 4.3 & $0-28.4$ & 0.0 \\
\hline OTA & & $82.9 \%$ & 0.36 & 0.29 & $0-1.76$ & 0.35 \\
\hline AFB1 & & $22.9 \%$ & 0.01 & 0.05 & $0-0.73$ & 0.00 \\
\hline AFM1 & & $50.2 \%$ & 0.11 & 0.18 & $0-1.91$ & 0.11 \\
\hline ZEA & & $5.4 \%$ & 0.1 & 0.4 & $0-3.9$ & 0.0 \\
\hline $\mathrm{DON}$ & & $19.5 \%$ & 1.0 & 3.6 & $0-27.9$ & 0.0 \\
\hline DOM1 & & $13.1 \%$ & 0.3 & 1.1 & $0-12.7$ & 0.0 \\
\hline FB1 & & $13.7 \%$ & 0.2 & 0.7 & $0-5.6$ & 0.0 \\
\hline Autistic Group & 160 & & & & & \\
\hline GLIO & & $23.0 \%$ & 1.8 & 4.5 & $0-28.4$ & 0.0 \\
\hline OTA & & $85.1 \%$ & 0.39 & 0.31 & $0-1.76$ & 0.35 \\
\hline AFB1 & & $24.0 \%$ & 0.01 & 0.06 & $0-0.73$ & 0.00 \\
\hline AFM1 & & $53.2 \%$ & 0.12 & 0.20 & $0-1.91$ & 0.11 \\
\hline ZEA & & $5.2 \%$ & 0.1 & 0.4 & $0-3.9$ & 0.00 \\
\hline $\mathrm{DON}$ & & $19.5 \%$ & 1.2 & 3.9 & $0-27.9$ & 0.0 \\
\hline DOM1 & & $12.9 \%$ & 0.3 & 1.2 & $0-12.7$ & 0.0 \\
\hline FB1 & & $17.5 \%$ & 0.3 & 0.7 & $0-5.6$ & 0.0 \\
\hline Siblings & 35 & & & & & \\
\hline GLIO & & $14.3 \%$ & 0.6 & 1.8 & $0-5.6$ & 0.0 \\
\hline OTA & & $77.1 \%$ & 0.27 & 0.21 & $0-0.79$ & 0.29 \\
\hline AFB1 & & $25.7 \%$ & 0.002 & 0.00 & $0-0.01$ & 0.00 \\
\hline AFM1 & & $45.7 \%$ & 0.07 & 0.10 & $0-0.36$ & 0.00 \\
\hline ZEA & & $8.6 \%$ & 0.1 & 0.2 & $0-1.2$ & 0.0 \\
\hline $\mathrm{DON}$ & & $22.9 \%$ & 0.5 & 0.9 & $0-2.3$ & 0.0 \\
\hline DOM1 & & $17.1 \%$ & 0.3 & 0.6 & $0-1.6$ & 0.0 \\
\hline FB1 & & $2.9 \%$ & 0.04 & 0.3 & $0-1.5$ & 0.0 \\
\hline
\end{tabular}


Table 5. Cont.

\begin{tabular}{ccccccc}
\hline Group & Observations & \% Positive & Mean $(\mathbf{n g} / \mathbf{m L})$ & Std Dev (ng/mL) & Min-Max (ng/mL) & Median $(\mathbf{n g} / \mathbf{m L})$ \\
\hline Non-Parental & $\mathbf{1 8}$ & & & & & \\
\hline GLIO & & $18.8 \%$ & 10.3 & 33.3 & $0-1$ & 0.0 \\
OTA & $75.0 \%$ & 0.28 & 0.2 & $0-0.61$ & 0.01 \\
AFB1 & $6.3 \%$ & 0.00 & 0.002 & 0.11 & $0-0.33$ & 0.00 \\
AFM1 & $31.3 \%$ & 0.06 & 0.0 & $0-0.0$ & 0.00 \\
ZEA & $0.0 \%$ & 0.00 & 2.8 & $0-11.0$ & 0.0 \\
DON & $12.5 \%$ & 0.8 & 0.3 & $0-1.4$ & 0.0 \\
DOM1 & $6.3 \%$ & 0.1 & 0.0 & $0-0.0$ & 0.0 \\
FB1 & $0.0 \%$ & 0.0 & & \\
\hline
\end{tabular}

* Due to the low amount of samples collected from some children, it was not always possible to carry out all the analyses planned for the study.

\subsubsection{Statistical Analysis}

The hypothesis of normality distribution (Shapiro-Wilk test) was refused, thus non-parametrical tests (which do not imply any distribution assumption) were used for the statistical treatment. The possible differences between concentration levels of mycotoxins in autistic children and control groups (either considered as a unique control or split into sibling and non-parental groups) were explored by a Wilcoxon rank-sum test.

Sub-groups with specific co-morbidity were also clustered as described in Table 6.

Table 6. Sub-groups of ASD patients with and without a specific comorbidity.

\begin{tabular}{cc}
\hline Sub-Groups with a Specific Co-Morbidity & Sub-Groups without Co-Morbidity \\
\hline SG1+ Patients with IgG allergenic values $>20 \mathrm{mg} / \mathrm{L}$ & SG1 - Patients without IgG allergenic values $\leq 20 \mathrm{mg} / \mathrm{L}$ \\
SG2+ Patients with gastrointestinal problems & SG2 - Patients without gastrointestinal problems \\
SG3+ Patients with verbal issue & SG3- Patients without verbal issue \\
SG4+ Patients with regressive autism & SG3- Patients without regressive autism \\
\hline
\end{tabular}

Finally, two more sub-groups were defined: (1) ASD paired sub-group (sub-group of autistic children with one brother/sister in the control group) and (2) sibling paired sub-group (sub-group of siblings of autistic children). For the statistical analysis, taking into consideration the dependence, the Wilcoxon signed-rank test was applied.

In Table 7, all differences between autistic children and controls or sub-groups of autistic children that showed a $p \leq 0.050$ for mycotoxin content in urine (_u) or serum (_s) are summarised.

Table 7. Differences statistically significant in mycotoxin concentration (in urine,_u and serum,_s) between groups and sub-groups.

\begin{tabular}{|c|c|c|c|c|c|c|c|c|}
\hline URINE & GLIO_s & OTA_u & OTA_s & AFB1_u & AFM1_s & FB1_u & DON_u & DOM1_u \\
\hline Autistic vs. control (all) & - & - & $p=0.0141$ & - & $p=0.0072$ & - & $p=0.0141$ & $p=0.0259$ \\
\hline Autistic vs. siblings & - & - & $p=0.0261$ & - & $p=0.0262$ & - & $p=0.0185$ & $p=0.0259$ \\
\hline Autistic vs. siblings paired & - & $p=0.0272$ & $p=0.0409$ & - & - & - & $p=0.0305$ & - \\
\hline $\mathrm{SG} 1+($ A. nidulans $)$ & - & - & - & - & $p=0.0457$ & - & - & - \\
\hline $\mathrm{SG} 1+($ A. flavus $)$ & $p=0.0169$ & - & - & - & - & - & - & - \\
\hline $\mathrm{SG} 1+(\mathrm{C}$. Albicans $)$ & - & $p=0.0169$ & - & $p=0.0462$ & - & $p=0.0126$ & - & $p=0.0487$ \\
\hline
\end{tabular}

Urine. DON and DOM1 levels in autistic children showed mean values statistically different when compared with levels of the control group considered as a whole (sibling and non-parental together) ( $p=0.014$ and $p=0.0259$, respectively); likewise, mean levels of DON and DOM1 were statistically different when compared with siblings ( $p=0.0185$ and $p=0.259$, respectively).

When GLIO levels in the autistic group were stratified for age, a statistical difference was found $(p=0.0127)$. 
By dividing the autistic children group in SG1+ and SG1-, the following differences were found: OTA levels were statistically different for Candida albicans $(p=0.0169)$, Fusarium moniliforme $(p=0.0075)$; AFB1 for C. albicans ( $p=0.0462)$; DOM1 for C. albicans $(p=0.0487)$ and FB1 for C. albicans $(p=0.0126)$.

The same analyses of the differences were applied to the subgroup of autistic children with a subgroup of brothers or sisters in the sibling group. Statistical significance was found in the difference of DON levels $(p=0.0305)$ and OTA levels $(p=0.0272)$.

To assess the relationship between mycotoxin level in urine and the variables, a Spearman's rank correlation coefficient ( $\rho$ ) was calculated. Negative and positive correlation was found and the following couples with $p$-values $<0.050$ were considered as a significant result: GLIO and A. flavus ( $\rho$ s $=-0.2631 p=0.0096) ; \mathrm{FB} 1$ and $A$. clavatus $(\rho s=-0.2046 p=0.0455)$; AFB1 and wheat $(\rho s=0.2188$ $p=0.0322)$; AFB1 and gluten ( $\rho s=0.2485 p=0.0061)$; DON and A. fumigatus ( $\rho s=-0.2777 p=0.0061)$.

Serum. OTA and AFM1 levels in autistic children showed mean values statistically different when compared with control group considered as a whole (sibling and non-parental together) $(p=0.0141$ and $p=0.0072$, respectively); differences were also found when they were compared with sibling control group (non-paired) ( $p=0.0261$ and $p=0.0262$, respectively).

When mycotoxin levels in the autistic group were stratified for age, OTA and DOM1 showed a statistical difference ( $p=0.0376$ and $p=0.0362$, respectively).

By dividing the autistic children group for co-morbidity, SG1+ and SG1-, the following differences were found: GLIO levels were statistically different for A. flavus $(p=0.0169)$; OTA for wheat $(p=0.0212)$; AFM1 for $A$. nidulans ( $p=0.0457)$.

Differences of AFM1 mean levels resulted statistically different $(p=0.0385)$ also in SG4+ and SG4-.

The analyses of the differences were performed to a subgroup of autistic children, which had a brother and/or sister in the sibling group and with a subgroup of brothers or sisters (by applying a Wilcoxon signed-rank test). Statistical significance was found for OTA $(p=0.0409)$.

To assess the relationship between mycotoxin level in urine and serum and the variables, a Spearman's rank correlation coefficient was calculated. Negative and positive correlation was found as reported in Table 8, where the $\rho$ s obtained with $p$-values $\leq 0.050$ are reported. GLIO and A. flavus ( $\rho s=0.2258 p=0.0270$ ); OTA and A. fumigatus ( $\rho s=-0.2466 p=0.0154$ ); AFB1 and A. oryzae ( $\rho s=-0.2232 p=0.0288)$; AFB1 and A. clavatus ( $\rho s=-0.2230 p=0.0290)$.

Table 8. Correlations between mycotoxin levels and variables (IgGs) in autistic group. Values of $\rho$ s for $p \leq 0.050$ are reported.

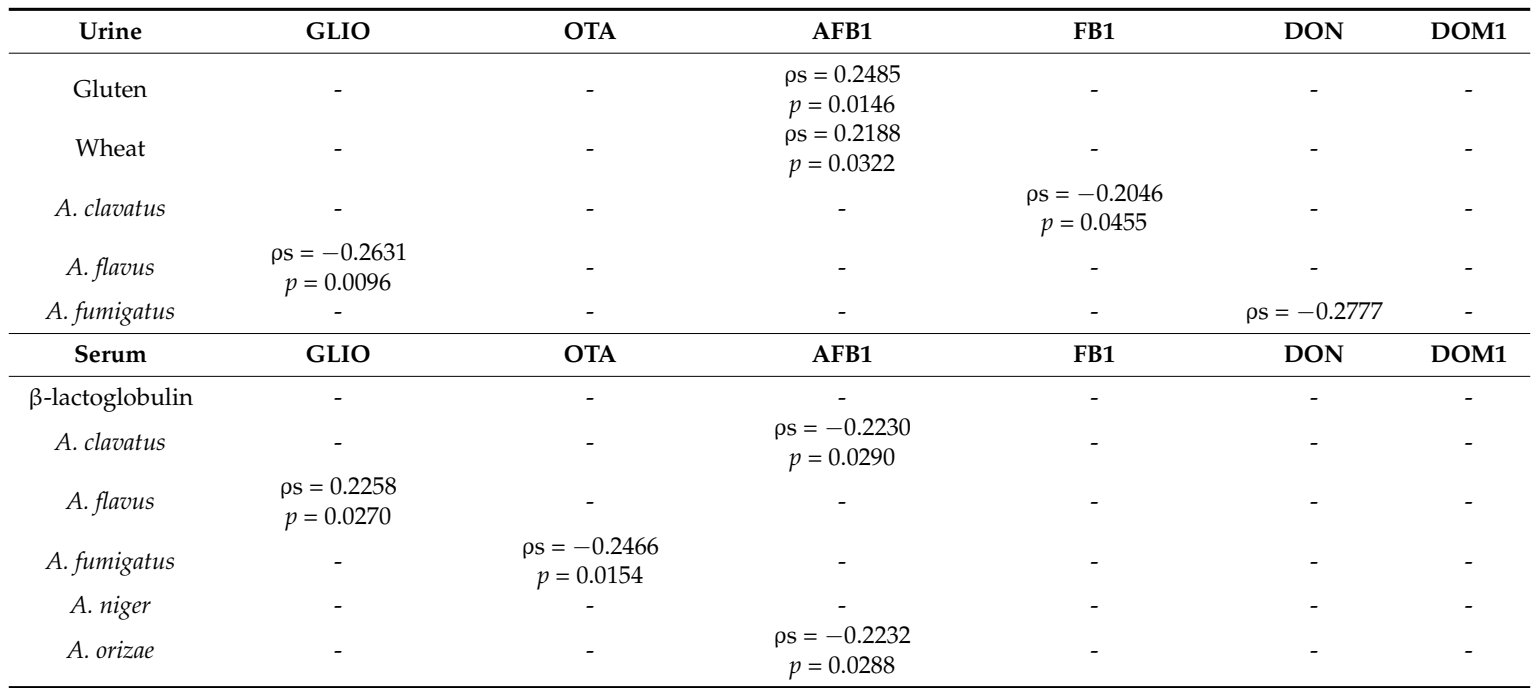




\subsection{Clinical Parameters and Cytokines Analyses}

The quantitative analysis of several serum cytokines and chemokines indicated some significant differences between healthy control and autistic children. Therefore, the statistical comparison was carried out and results have been summarized in Table 9. All correlations are positive and values of Spearman's rank correlation coefficient result from weak to moderate.

Table 9. Correlations between mycotoxin levels and cytokine/chemokines in autistic group. Values of $\rho$ s are reported for all correlations that gave $p \leq 0.050$.

\begin{tabular}{cccccc}
\hline \multirow{2}{*}{ Cytokines/Chemokines } & \multicolumn{3}{c}{ Serum } & \multicolumn{2}{c}{ Urine } \\
\cline { 2 - 6 } & OTA & AFM1 & DON & AFB1 & ZEA \\
\hline PDGF-BB & - & 0.3209 & 0.2141 & - & - \\
Il-1ra & 0.2296 & - & - & - & - \\
Il-4 & 0.2219 & - & - & - & - \\
Il-5 & - & 0.2859 & 0.2519 & - & - \\
Il-7 & - & 0.3546 & 0.2484 & 0.2107 & 0.2343 \\
Il-12 & - & 0.2498 & - & - & - \\
Il-13 & - & 0.2220 & - & - & - \\
IFN-g & 0.2195 & 0.3235 & 0.2357 & - & - \\
EOT & 0.1810 & 0.3141 & - & - & - \\
TNF-alfa & 0.1730 & 0.2389 & - & - & - \\
RANTES & - & - & 0.2348 & - & - \\
\hline
\end{tabular}

\section{Discussion}

\subsection{Occurrence}

This study presents a multi-mycotoxin method suitable to detect a group of mycotoxins in urine and serum. Some $\mathrm{R}_{\mathrm{A}}$ in serum was below $60 \%$ with high suppression signal but, considering the precision data values ( $\leq 23 \%$ ), these results were considered reliable, especially considering the advantage of a multi-mycotoxin detection (see Table 3).

Taking into consideration the data as a whole, the present work constitutes a densely populated dataset and a precious source of information about possible biomarkers of exposure to mycotoxins in Italian children.

OTA is the mycotoxin with higher numbers of positive results both in urine and serum $(74.8 \%$ and $82.9 \%$ of positive samples, respectively), followed by GLIO (71.6\%), AFB1 (66.2\%), AFM1 (62.6\%) in urine samples and by AFM1 (50.2\%) and AFB1 (22.9\%) in serum samples.

As for levels of OTA, DON, AFB1 and AFM1 found in urine, these are in line with other data in literature for children or adults [24-26].

More specifically, OTA mean values found in this study in autistic children were in agreement with the mean values found in a pilot study that was conducted in a smaller autistic children group $(n=52)$ and controls $(n=58)$ [27] $(0.06 \mathrm{ng} / \mathrm{mL}$ in urine and $0.40 \mathrm{ng} / \mathrm{mL}$ in serum). OTA positivity seems to be of high importance, since it contaminates most of food products especially those consumed by children, such as cereal-derived products and chocolate.

Regarding DON and DOM1, our results are slightly different from those presented in the experimental study of DON biomarkers in urine performed by the European Food Safety Authority (EFSA) [28] to assess the exposure to DON in the European Union (EU) population. In that study, the Italian group of children and adolescents showed almost $100 \%$ of subjects positive to DON (among 80 to $100 \%, 0.43-75.9 \mathrm{ng} / \mathrm{mL}$ range, mean) and $0 \%$ of positive results to DOM1 for the same group. Here, considering DON, about $40 \%$ of urine samples were above the LoQ with mean value only of $1.4 \mathrm{ng} / \mathrm{mL}$, while DOM1 resulted positive on the $27 \%$ of the samples with a mean value of $8.4 \mathrm{ng} / \mathrm{mL}$. 
AFB1 and AFM1 showed a substantial number of positive results both in urine and serum. Considering their toxicity (AFB1 is carcinogenic and genotoxic, classified in group 1 by IARC [29]) this constitutes a concern of exposure.

GLIO values found in the present study confirmed percentages values of positive results higher in urine than in serum, though mean levels were slightly lower than those of the pilot study [27].

FB1 was tested in urine and serum, resulting positive in a small number of samples ( $44 \%$ and $13.7 \%$ in urine samples and in serum samples, respectively) with low levels, very close to LoQ values.

As regards the exposure to ZEA, it was undetectable in $50 \%$ of urines. Conversely on what was found in [27], where ZEA, $\alpha$-ZEA and $\beta$-ZEA were all not detected, positive values for ZEA in urine were found close to LoQ (range $1.6-6.5 \mathrm{ng} / \mathrm{mL}$ ). The lower LoQ values $(1.6 \mathrm{ng} / \mathrm{mL}$ in the present method vs. $5 \mathrm{ng} / \mathrm{mL}$ in De Santis et al. [27]) have increased the detectability of positive results, when present. This also explains the higher number of positive results found in the present study in comparison with the ones published by Duringer [30]. In fact, Duringer conducted a screening for urinary mycotoxins in 25 autistic patients and 29 healthy controls and found one positive result for 3-Acetyldeoxynivalenol. Indeed, they tested 87 urinary mycotoxins via liquid chromatography-tandem mass spectrometry, presenting a good screening. To note, as they also highlighted, they used a dilute and shoot sample preparation with high LoQs (almost 10 times higher in comparison with those reported here), thus, explaining the reported differences.

\subsection{Statistical Associations}

As regards the statistical analyses that were conducted, we focused on the comparison between the patients group and the control group to find out possible differences and associations between mycotoxins and other variables in ASD. In this respect, we excluded comparisons with those mycotoxins that had less than 15\% of positive results (namely, ZEA and DOM1 and FB1 in serum samples) in order to avoid misreported outputs because of the number of zeros or values below LoQs.

Statistically significant differences are shown in Table 7 . These differences are not intended to explain the pathobiology of the disorder, but rather to give information on the presence of possible environmental risk factors that may act as perturbative agents involved in the gene-environment interaction, the more accredited hypothesis for ASD onset.

Differences of OTA, AFM1 levels in serum and DON and DOM1 levels in urine were found between autistics and the control group (siblings and non-parental, summed) and (excepting DOM1) between autistic and siblings group. Furthermore, OTA and DON in urine confirmed statistically significant differences when autistic children were paired and compared with their brother or sister.

These differences in the levels of exposure in subjects from the same family that presumably have the same diet, should be deeply explored as the same toxins can have effects of different extent depending on individual predisposition. According to Smith [6], the intestine is a major target for the toxic effects of AFs, DON and FBs, because of their effect in the impairment on nutrient uptakes and intestinal pathology. Altered intestinal architecture can result in a loss of enzymes, leading to malabsorption of a variety of nutrients. AFs, DON and FBs may therefore share a convergent pathway in which mucosal damage can lead to impaired nutrient absorption and/or increased intestinal permeability [6]. Thus, to be clarified is the perturbative effect of chronic exposure to these toxins by ASD children with GI issues, which can favour or worsen persistent damages, and/or with microbioma-brain-gut signalling that may play a role in brain development or result in neuroplastic and functional alterations [31].

To explore the relationship between food- or mould-specific IgGs levels of ASD patients and mycotoxins, two subgroups were defined: one with IgGs levels $>20 \mathrm{mg} / \mathrm{L}$ and one with IgG values $\leq 20$ $\mathrm{mg} / \mathrm{L}$. Some mycotoxins highlighted differences that were statistically significant especially among specific fungal IgG groups as reported in Table 7.

SG1+ and SG1 - were defined for food-specific IgGs and the only difference regarded OTA levels in serum in SG1+ for wheat group. 
Correlations, by assessing Spearman's rho, were run to determine relationship between couples of mycotoxins and IgGs values in the autistic group (Table 8). Weak, positive and negative monotonic correlations were determined: A. clavatus and AFB1 and FB1; A. flavus and GLIO; A. fumigatus and OTA and FB1; $A$. niger and DOM1; A. orizae and AFB1; gluten and AFB1; wheat and AFB1; $\beta$-lactoglobulin and DOM1.

GI issues and associated symptoms are commonly reported in individuals with ASD [32], and from large prospective well-controlled studies it was confirmed that GI problems, particularly constipation and diarrhoea, affect children with ASD and developmental delay far more often than children with typical development [33]. Our results also evidenced that recurrent vomit, aerophagia, diarrhoea and intestinal anomalies are more likely to occur in autistic children.

It is thus plausible to hypothesize that the toxic effect of mycotoxins as well as their biological consequences may contribute to the aggravation of the general conditions of patients including leaky gut for which the increased permeability, hypothesizing barrier defects and affecting the mucosal immune system, lead to changes in gut flora [34].

This association among mycotoxins and autism was explored by a study of Duringer and co-workers [30]. Due to the low number of positive results (equal in both groups), they could not find any kind of difference or correlation between ASD and the control group. Indeed, their study has a smaller sample size ( 25 autistic children and 29 children as controls), a lack of sensitivity for the sample preparation (LoQ for deoxynivalenol was $50 \mu \mathrm{g} / \mathrm{L}$ ) and no genetic test was previously performed on patients so the inclusion of subjects with causative genetic variations cannot be excluded.

The statistical differences of serum levels of cytokines reported in Table 9 indicated interesting and significant correlations between mycotoxin levels and cytokine/chemokines in autistic group. The cytokine/chemokines correlated to mycotoxin levels are known to be involved in several pathological conditions, in particular platelet derived growth factor (PDGF) and tumor necrosis factor (TNF)-alpha are considered important pro-inflammatory signals, IL-13 and eotaxin are involved in inflammation and allergic conditions, as well as IL-7 and IL-12 in immunity responses. Alterations of plasmatic levels of some of the cytokines reported in this study were also described in other studies [35,36]. The cytokine levels differences suggest that exposure to mycotoxins may represent a portion of a complex scenario involving both innate and adaptive immune responses. Due to the reported co-morbidity of other diseases with ASD (just as an example the above reported GI issues) [32], this serum quantification of cytokines confirms a key role of immune system in ASD children pathology. Nevertheless, it is not yet clear whether the pro-inflammatory phenotype observed coupled to the GI symptoms may represent the effect of a more general immune system deregulation or whether they play a role in ASD pathogenesis. Further, it should also be taken into account that significant alterations of microbiota (including bacteria and fungi) and GI mucosa in ASD children (strongly suggested by occurrence of diarrhoea and intestinal anomalies) might facilitate the entry of toxins and/or antigens into the blood stream, as well as mycotoxins, and may mediate intestinal damage [6]. Several pathogenic bacteria strains, indeed, produce lipopolysaccharides that increase the intestinal tight junction permeability [37] as well as some other produce pore-forming toxins, protein exotoxins that induce pores formation in the cell membrane [38]. The increase of Candida genus, just found by Strati and collaborators in the gut of ASD children [19] further support the role of "leaky gut" in xenobiotic adsorption and consequent immune reactivity. Indeed, in animal model, Candida colonization of intestinal tract promotes the absorption and sensitization against food antigens [39]. Moreover, gut eubiosis (beneficial microbes) produces short chain fatty acids (SCFAs) that, in turn, increase the production of the tight junction proteins of brain-blood barrier (BBB). On the contrary, dysbiosis decreases SCFAs production that affects the integrity of BBB [18] and BBB permeability has already been described in ASD patients [40]. 


\subsection{Interventions}

Intestinal permeability plays a key role in food allergies and intolerances and in autistic children we have found a correlation between exposure to OTA, ZEA, AFB1, DOM1 regarding wheat, and gluten. The gluten-free/casein-free diet is a recurring finding in the literature with regard to autistic children, with alternate results. The tendency of ASD children to eat often the same foods can cause an overload in the intake of several substances and contaminants, such as mycotoxins. Also interesting is the detection of IgGs against fungal strains that denounce the contact with the fungus. We believe that in ASD children with GI problems and altered intestinal permeability, intolerances should be sought and, if positive, a rotation diet should be applied [41] together with probiotics, in order to improve their clinical issues.

\section{Experimental Section}

\subsection{Materials and Methods}

\subsubsection{Subject Enrolment: Inclusion Criteria}

A group of autistic patients $(n=172)$ and a control group $(n=61)$ were recruited by the Italian Research Hospital, E. Medea (Istituto di Ricovero e Cura a Carattere Scientifico, IRCCS), from two locations: Bosisio Parini (Lecco, Lombardia, Italy) and Ostuni (Brindisi, Puglia, Italy).

During the first contact with children and their families (or tutors), medical staff gave the presentation of the research project detailing context and aims. The medical staff interviewed all those who decided to participate. Children were tested with specific psychological tests; urine and serum samples were collected.

For autistic children, the inclusion criteria were the following: age in the range 2 to 12 years old, diagnosis of autistic spectrum disorder, with exclusion of other genetic syndromes (e.g., $\mathrm{X}$ fragile), epilepsy, or other medical disorders or neurological disturbances.

The Ethical Committee of IRCCS Eugenio Medea-La Nostra Famiglia, approved the study.

The autistic diagnosis was verified by a team of psychiatrics and psychologist applying the Autism Diagnostic Observation Scale 2 [42] and The Autism Diagnostic Interview [43]. The same medical team submitted other tests for the assessment of adaptive behaviour (Vineland Adaptive Behaviour Scale) [44], for the measure of the breadth of repetitive behaviour (Repetitive Behavior Scale-Revised) [45] or Griffiths Mental Development Scales [46], for the assessment of intelligence scale (preschool and primary scale Wechsler intelligence scale) $[47,48]$, and for the identification of problem behaviour (the Child Behavior Check List, CBCL) [49,50].

The control group was composed of a subgroup of siblings $(n=36)$ and a subgroup of non-parental $(n=25)$, which did not have any parental relationship with the autistic group. The CBCL $[49,50]$ was applied to the control group in order to exclude possible pathologies.

Besides, medical history and food habits were recorded through ad hoc questionnaires. A medical general questionnaire was completed by the parents to acquire info on the general status of the mother (before and during pregnancy) and of the children (from the birth to that moment), on the ingestion of drugs; the presence of GI disorders, of food allergies or intolerances, and special dietary needs in children and adolescents were assessed.

\subsubsection{Specimen Collection}

All those who decided to participate were informed on how to collect first morning urine samples on the provided sterile tubes. The children blood sampling date and the children urine samples consignment was agreed with the parents.

For the analyses, an aliquot $(50 \mathrm{~mL})$ of urine was delivered, while the blood sample was taken by the medical staff. Biological samples were stored at $-20{ }^{\circ} \mathrm{C}$ until delivery to the Italian National Institute for Health laboratories (ISS, Rome), where they were stored at $-20{ }^{\circ} \mathrm{C}$ until analyses. 


\subsubsection{Mycotoxin Analysis}

Analyses of AFB1 and AFM1, OTA, DON, DOM1, ZEA, FB1 and GLIO were performed by GMO and Mycotoxin Unit, Italian National Institute for Health.

Aflatoxin B1, AFM1, OTA, DON, DOM1, ZEA, FB1 and GLIO were tested in serum and urine with method previously validated for precision and trueness performances.

The principle of the methods for the mycotoxin analyses in serum and urine are briefly reported.

Mycotoxins from serum: the extraction procedure was performed on $1 \mathrm{~mL}$ of sample to which $18 \mu \mathrm{L}$ of pronase (Sigma-Aldrich, Saint Louis, MO, USA) and $1 \mathrm{~mL}$ of phosphate buffer were added prior to the incubation step (overnight, $37^{\circ} \mathrm{C}$ ). The sample was mixed and vortexed with $2 \mathrm{~mL}$ of ethyl acetate $1 \%$ formic acid, vigorously shaken in a laboratory shaker for $30 \mathrm{~min}$ and then centrifuged $15 \mathrm{~min}$ at $4000 \mathrm{rpm}$. The organic phase was transferred to a clean vial and gently dried under a gentle $\mathrm{N}_{2}$ stream. After adding and mixing the aqueous phase with $1 \mathrm{~mL}$ of acetonitrile, $1.6 \mathrm{~g}$ of QuEChERS (DisQUE, Waters, Milford, MA, USA) were also added. The sample, vigorously mixed (3 min vortex), was then centrifuged 15 minutes at $4000 \mathrm{rpm}$. The recovered supernatant was added to the same clean vial and gently dried under a gentle $\mathrm{N}_{2}$ stream.

Mycotoxins from urine: the extraction procedure was performed on $3 \mathrm{~mL}$ of sample to which $3 \mathrm{~mL}$ of ethyl acetate $1 \%$ formic acid was added. The sample was vigorously shaken in a laboratory shaker for $30 \mathrm{~min}$ and then centrifuged for $15 \mathrm{~min}$ at $4000 \mathrm{rpm}$. The organic phase was transferred to a clean vial and gently dried under $\mathrm{N}_{2}$. After adding and mixing the aqueous phase with $3 \mathrm{~mL}$ of acetonitrile, $2.1 \mathrm{~g}$ di QuEChERS (DisQUE, Waters, Milford, MA, USA) were also added. The sample was vigorously shaken for $10 \mathrm{~min}$, and then centrifuged for $15 \mathrm{~min}$ at $4000 \mathrm{rpm}$. The recovered supernatant was added to the same clean vial and gently dried under a gentle $\mathrm{N}_{2}$ stream.

Both dried serum and urine samples were reconstituted with $0.3 \mathrm{~mL}$ of injection solvent $\left(\mathrm{MeOH} / \mathrm{H}_{2} \mathrm{O} 3 \%\right.$ formic acid and $5 \mathrm{mM}$ ammonium formiate, 50/50, $\left.v / v\right)$. The samples were then filtered on $0.22 \mu \mathrm{m}$ membrane disc filters polyvinylidene difluoride (PVDF) before injection.

The mycotoxins were detected on an Acquity UPLC, Quattro Premier LC-MS/MS system (Waters Corporation, Milford, MA, USA), via electrospray ionization. The chromatographic separation was obtained in UPLC with a binary gradient elution mode, at $40{ }^{\circ} \mathrm{C}$, on a $\mathrm{C} 18$ Kinetex column (Biphenil $100 \mathrm{~mm}, 2.6 \mu \mathrm{m}, 50 \times 3 \mathrm{~mm}$; Phenomenex, Torrance, CA, USA) and an in-line filter unit.

Both eluent $A$ and eluent $B$, composing the mobile phases, contained $5 \mathrm{mM}$ ammonium formiate and 3\% formic acid and were made up of acetonitrile/water 50/50 $(v / v$; eluent $A)$ and 50/50 methanol/water $(v / v$; eluent B). After an initial time of $2.5 \mathrm{~min}$ at $95 \%$ eluent $\mathrm{A}$ and $5 \%$ eluent $\mathrm{B}$, the proportion of B was increased to $25 \%$ (75\% eluent A) for further $1.5 \mathrm{~min}$, to $60 \%$ ( $40 \%$ eluent A) for further $2 \mathrm{~min}$, to $90 \%(10 \%$ eluent A) for further $1.5 \mathrm{~min}$, and to $100 \%$ for further $1.5 \mathrm{~min}$. After that, the column was re-equilibrated for $3.5 \mathrm{~min}$ at $95 \%$ eluent $\mathrm{A}$ and $5 \%$ eluent $\mathrm{B}$. The flow rate was $0.3 \mathrm{~mL} / \mathrm{min}$.

Electro Spray Ionisation (ESI)-MS/MS was performed in multiple reaction monitoring (MRM) mode in positive polarity with the following settings: a capillary voltage of $3.5 \mathrm{kV}$, a desolvation temperature of $350^{\circ} \mathrm{C}$, a desolvation gas flow of $600 \mathrm{~L} / \mathrm{h}$, a cone gas flow of $100 \mathrm{~L} / \mathrm{h}$ and nebuliser gas fully open, all nitrogen. Dwell time was $0.02 \mathrm{~s}$. The collision gas was argon at $3.510^{-3} \mathrm{~atm}$ on a pressure regulator. Ionisation and MS/MS collision energy settings were optimised infusing separate mycotoxin solutions $(1-2 \mathrm{mg} / \mathrm{mL}$ ) at a flow-rate of $10 \mathrm{~mL} / \mathrm{min}$. For each mycotoxin, the parent and two daughter ions were recorded; the optimized conditions are listed in Table 10. Masslynx and Quanlynx softwares were used for data acquisition and processing.

Primary standards (Biopure by Romer Labs ${ }^{\circledR}$ (Getzersdorf, Austria) and Sigma-Aldrich ${ }^{\circledR}$, Saint Louis, MO, USA) were diluted in appropriate working solutions for the preparation of neat standard calibration curves. Reagents Liquid Chromatography (LC) gradient grade methanol and acetonitrile, as well as MS grade ammonium formiate and formic acid were purchased from Sigma-Aldrich ${ }^{\circledR}$. 
Table 10. MS/MS conditions for detection of target analytes.

\begin{tabular}{|c|c|c|c|c|c|}
\hline Mycotoxin & $\begin{array}{c}\text { Parent }[\mathbf{M}-\mathrm{H}]^{+} \\
(\mathrm{m} / \mathrm{z})\end{array}$ & $\begin{array}{c}\text { Cone Voltage } \\
(V)\end{array}$ & $\begin{array}{c}\text { Quantifier/Q } \\
\text { Ion }(m / z)\end{array}$ & $\begin{array}{l}\text { Collision } \\
\text { Energy (eV) }\end{array}$ & $\begin{array}{c}\text { Retention } \\
\text { Time (min) }\end{array}$ \\
\hline GLIO & 327.3 & 12 & $263.3 / 245.3$ & $8 / 18$ & 6.1 \\
\hline OTA & 404.1 & 20 & $238.9 / 341.0$ & $20 / 20$ & 7.5 \\
\hline AFB1 & 313.3 & 30 & $285.3 / 128.1$ & $22 / 22$ & 8.4 \\
\hline AFM1 & 329.3 & 30 & $273.3 / 259.3$ & $22 / 22$ & 7.1 \\
\hline ZEA & 319.2 & 10 & $283.1 / 301.1$ & $10 / 10$ & 6.9 \\
\hline DON & 297.3 & 22 & $249.5 / 203.5$ & $10 / 15$ & 4.0 \\
\hline DOM1 & 281.2 & 15 & $108.7 / 215.1$ & $15 / 10$ & 4.4 \\
\hline FB1 & 723.4 & 50 & $335.5 / 353.5$ & $40 / 30$ & 5.7 \\
\hline
\end{tabular}

\subsubsection{Method Validation}

The performance characteristics of the method for urine and serum for the seven mycotoxins were obtained with replicated $(n=3)$ spiking experiments carried out at five concentration levels in the relative concentrations of 1:2:3:4:6 in the final diluted extracts. Neat standard calibration curves for all the mycotoxins were prepared by dilution of appropriate amounts of the final working solution with methanol: water $0.3 \%$ formic acid, $5 \mathrm{mM}$ ammonium formiate $(50 / 50, v / v)$ at levels close to those used in spiked samples. For the assessment of matrix effects and extraction efficiency (recovery), the diluted blank urine and serum extracts were fortified at the concentration range matching the neat standard calibration curve.

For the validation of the method, the whole procedure was carried out in triplicate at different concentration levels: 5 levels for urine (relative concentrations of 1:2:5:14:50) and 9 levels for serum (relative concentration of 1:1.5:3:4.5:6.5:10:15:20:30) were performed. From these levels, the spiked sample curve was obtained. The validation protocol also included the testing of blank extracts (performed in triplicate) spiked after extraction at concentration levels comparable with the matrix (urine or serum); from these levels, the spiked extract curve was obtained. Moreover, neat standard calibration curves for each analyte were run by injecting 5 levels of analyte (mixed standards with relative concentrations of 1:3:6:15:30).

The apparent recovery or absolute recovery of the method $\left(\mathrm{R}_{\mathrm{A}}\right)$, the signal suppression/enhancement (SSE) due to matrix effects and the recoveries as extraction efficiency $\left(\mathrm{R}_{\mathrm{E}}\right)$ were evaluated on the basis of the obtained slopes of the 3 curves constructed by plotting the signal intensity versus the analyte concentration [16]. Equations (1)-(3) below describe how SSE, $R_{A}$ and $R_{E}$ were calculated.

$$
\begin{gathered}
\text { SSE }=100 \times \mathrm{SLOPE}_{\text {SPIKED BLANK CALIB CURVE }} / \mathrm{SLOPE}_{\text {NEAT STANDARD CALIB CURVE }} \\
\mathrm{R}_{\mathrm{A}}=100 \times \mathrm{SLOPE}_{\text {SPIKED CALIB CURVE }} / \mathrm{SLOPE}_{\text {NEAT STANDARD CALIB CURVE }} \\
\mathrm{R}_{\mathrm{E}}=100 \times \mathrm{R}_{\mathrm{A}} / \mathrm{SSE}
\end{gathered}
$$

Limits of quantifications (LoQs) were calculated based on signal-to-noise (S/N) ratio of 10/1.

\subsubsection{Clinical Parameters and Cytokine Analyses}

Measurement of food- and mould-specific allergological IgG (Table 11) were performed in IRCCS E. Medea. Cytokine analyses were performed by the laboratory of the Facility for Complex Protein Mixture (CPM) Analysis at ISS, Rome.

Allergological food- and mould-specific IgG (Table 10) were assessed by Immulite $2000 \mathrm{XPi}$ automated system with an allergen-specific IgG test on a solid-phase, enzyme-labelled chemiluminescent immunometric assay. Chemiluminescent signal was measured as proportioned to the bound enzyme (Standard World Health Organization (WHO) WHO 1st International Reference Preparations (IRP) 67/86). The analytical sensitivity of the assay is $1 \mathrm{mg} / \mathrm{L}$ (reportable range of the test: 2-200 mg/L-WHO 1st IRP 67/86). Regarding analytical specificity, the endogenous 
interference studies showed no significant effect on patient results with the following substance concentrations: bilirubin up to $200 \mathrm{mg} / \mathrm{L}$, hemoglobin up to $522 \mathrm{mg} / \mathrm{dL}$, and triglycerides up to 3000 mg/dL (IMMULITE 2000 systems Allergen Specific IgG instructions, Siemens Healthineers, Malvern, PA, USA). The detection antibody for the assay is a murine monoclonal anti-human IgG antibody that is specific to total human IgG.

Table 11. List of variables and their description.

\begin{tabular}{ll}
\hline \multicolumn{1}{c}{ Variables } & \multicolumn{1}{c}{ Description } \\
\hline Location & Bosisio Parini (Lecco, Lombardia, Italy) Ostuni (Brindisi, Puglia, Italy) \\
\hline Symptom pattern & Verbal/Non-verbal \\
\hline \multirow{4}{*}{ Questionnaire Information } & Age \\
& Gex \\
& Painful bowel, Gastroenteritis, Aerophagia, Abdominal bloating, Lack of \\
& appetite, Intestinal infections, Intestinal anomalies) \\
\hline & Food-specific allergological IgG (wheat, gluten, casein, egg yolk, egg white, \\
& alfa-lactoalbumin, beta-lactoglobulin) \\
& Mould-specific allergological IgG (Aspergillus fumigatus, A. niger, A. oryzae, \\
Clinical trial parameters & A. terreus, A. nidulans, A. flavus, A. clavatus, Candida albicans, Fusarium \\
& moniliforme) \\
\hline Cytokines & PDGF-BB, Il-1ra, Il-4, Il-5, Il-7, Il-12, Il-13, IFN-g, EOT, TNF-alfa, RANTES \\
\hline
\end{tabular}

Cytokines and growth factors in sera from children affected by ASD and controls were measured by Luminex technology using a Bio-Plex Pro Human Cytokine 27-plex panel, (Bio-Rad Laboratories, Hercules, CA, USA) for the following different analytes (PDGF-BB, Il-1ra, Il-4, Il-5, Il-7, Il-12, Il-13, IFN-g, EOT, TNF-alfa, RANTES). The analysis was performed using $15 \mu \mathrm{L}$ of the serum as per manufacturer's instructions. After the incubation with antibodies-activated magnetic beads, samples were washed using a Bio-Plex Pro TM Station (Bio-Rad) as described [51], diluted and incubated with antibodies-activated magnetic beads then washed using a Bio-Plex Pro TM Station (Bio-Rad) according to the manufacturer's instructions. The quantification was carried out with a Bio-Plex Array Analyzer (X200, Bio-Rad) and analyzed with Bio-Plex Manager Software version 6.1. Results were expressed as $\mathrm{pg} / \mathrm{mL}$.

\subsubsection{Statistical Analysis and data Handling}

Shapiro-Wilk test was used to verify the distribution of the quantitative data. As the hypothesis of normality was refused, non-parametrical (which do not imply any distribution assumption) set of tests were used for the statistical treatment. More specifically, in order to assess the possible differences between concentration levels of mycotoxins in autistic children and control groups, the Wilcoxon rank-sum test (Mann-Whitney two-sample statistic [52,53]) was used. Where the collation of variables implied more than one distribution (Intelligent Quotients (IQs or age groups), Kruskal-Wallis test was used [54].

In order to reduce/control the genetic variability of the syndrome, two groups were defined: (1) a group of autistic children with one brother/sister in the control group and (2) a group of siblings of autistic children. For the statistical analysis, taking into consideration the dependence, the Wilcoxon signed-rank test was applied.

Lastly, to assess the correlation between mycotoxin levels and quantitative variables, a Spearman's rank correlation coefficient (or Spearman's rho) was used.

All tests were conducted with a level of significance of 5\%. All outputs are reported together with the level of statistical significance, $p$-value. Analyses were conducted by means of STATA14 software (Stata/IC 14.0, Copyright 1985-2015 StataCorp LP). 
As regards the data handling for the setting of the data set, depending on the nature (if quantitative or qualitative), variables were treated as follows:

1. Mycotoxins: quantitative, non-negative variable expressed as $\mathrm{ng} / \mathrm{mL}$. Values were assigned as follows:

- Value $=0 \mathrm{ng} / \mathrm{mL}$; when no analytical result derived from the analysis, thus no concentration could be derived;

- Value $=\mathrm{LoQ} / 2 \mathrm{ng} / \mathrm{mL}$; the half of the limit of quantification $(\mathrm{LoQ} / 2)$ was assigned to those analytical results that gave a value included between zero and the limit of quantification. These samples could not be quantified with the established precision of the method (below 23\%).

- Values = numerical value to all analytical results $\geq \mathrm{LoQ} \mu \mathrm{g} / \mathrm{mL}$; the value is expressed in $\mathrm{ng} / \mathrm{mL}$ rounded to the second figure after the comma for (DON, DOM1, FB1 and ZEA), and to the third figure after the comma for OTA, AFB1 and AFM1.

2. IgG quantitative, non-negative variable expresses ad $\mathrm{mg} / \mathrm{L}$. In addition to the quantity, a stratification was performed generating a binary on the basis of a threshold of $20 \mathrm{mg} / \mathrm{L}$ :

- Value " 0 " when the IgG value is $\leq 20 \mathrm{mg} / \mathrm{L}$;

- Value " 1 " when the IgG value is $>20 \mathrm{mg} / \mathrm{L}$

3. Cytokines: quantitative, non-negative variable: expressed as $\mathrm{pg} / \mathrm{mL}$.

4. The variables age, intelligence quotient, GI problems and verbal were stratified as follows:

- Age. This variable has been set defining the age at the moment of the sampling. The age was split in three classes: $2-4.5$ years old; $>4.5-7$ years old; $>7$ years old.

- Gastrointestinal Issues (GI). This is a dummy variable: presence of GI takes the value " 1 ", when one (or more) of the answers related to GI problems was positive, absence takes the value " 0 ", when all the answers related to GI problems were negative. It was derived on the basis of the answer given on the questionnaires on the presence or absence of the following: Vomit, Diarrhoea, Constipated, Stomach-aches, Painful bowel, Gastroenteritis, Aerophagia, Abdominal bloating, Lack of appetite, Intestinal infections, Intestinal anomalies.

- Verbal. This is a dummy variable: presence of verbal language skills takes the value " 1 ", absence takes the value " 0 ". The verbal language skill was derived from the tests submitted to the autistic patients.

- Regressive. This is a dummy variable: presence of regressive pattern takes value " 1 ", absence takes value " 0 ". The regressive pattern was derived from the tests submitted to the autistic patients.

\section{Conclusions}

Recent data indicate that in the last few decades there has been an alarming increase in childhood neurobehavioral disorders, for example from learning difficulties, attention deficit disorders, to the most severe forms such as mental retardation and autism, and the syndromes related to it. Such an increase in the last decades is partly due to greater sensitivity and improved diagnostic criteria but there is sufficient evidence to hypothesize a role for a number of environmental risk factors for the general population, and for children in particular, food being recognized as a priority exposure both for natural substances, such as mycotoxins, and other bioactive xenobiotics, such as phytoestrogens [55]. These xenobiotics can be internalized at various levels of the digestive process going from the flora intestinal bacterial, intestinal tissue itself until the liver. The health impact that mycotoxins have is well established and for this reason it is imperative to maintain the level of these toxic substances in food as low as possible. Our results show that ASD patients have a significant amount of several mycotoxins 
in their body fluids with respect to the controls, even with respect to their siblings that should share the same environment and food habits.

It is noteworthy that the mycotoxins effects on cell chemotaxis have been reported [56-58] as well as chemokine pathways and cell acidification involvement in endothelial and neurodevelopmental disorders [59-61] have been demonstrated. Our data reported support these findings and open new interesting perspectives connecting mycotoxins, chemokines and neurodevelopmental disorders.

Heterogeneous and variable phenotypes in autistic syndromes are framed in a geneenvironment-immune system complex grid and, even if any of the possible interrelationships into the triad has been elucidate yet, the multifactorial etiology is the only key that may explain this complexity [34]. This suggests that ASD patients exposed to the negative effects of these known xenobiotics can experience detrimental consequences, proposing mycotoxins as triggering factors for the impairment of medical co-morbidities in autistic children.

The association of mycotoxins with IGgs and cytokine/chemokines elicit to consider these xenobiotics among the perturbative agents involved in the gene-environment interaction in the attempt to elucidate the role of environmental risk factors in the pathogenesis for ASD onset.

This study opens the way to environmental xenobiotics to launch further relevant investigations that we intend to pursue to investigate the association between negative effects of mycotoxins on child health and ASD susceptibility.

Acknowledgments: This work has been supported by the Italian Ministry of Health (Targeted Research Funding in Public Health-Young Researchers; grant no. GR-2009-1570296); the technological support from the Facility for Complex Protein Mixture (CPM) Analysis at ISS (Rome), is gratefully acknowledged. Alessandra Mezzelani and Luciano Milanesi have been partially supported by Flagship project InterOmics (PB05); Anna Marabotti has been partially supported also by FARB-ORSA151138 and 161582, University of Salerno.

Author Contributions: Barbara De Santis: wrote the paper, analyzed the data, contributed to conceive, designed and overviewed the experiments; Maria Elisabetta Raggi: conceived and designed the experiments, performed the experiments; Giorgio Moretti: analyzed the data; Francesco Facchiano: analyzed the data and discussed the results; Alessandra Mezzelani: discussed the results and revised the manuscript; Arianna Bonfanti, Serena Camposeo: enrolled the children groups; interviewed and test the children. Laura Villa performed the ASD diagnoses; Alessandra Campioni, Stefania Rossi, Sabina Soricelli, Francesca Debegnach, Emanuela Gregori and Gabriele Moracci: performed the experiments; Francesca Ciceri: encoded all biological samples; Luciano Milanesi: discussed the results and revised the manuscript; Anna Marabotti: contributed reagents/materials/analysis tools, contributed to conceive and designed the experiments and revised the paper; Carlo Brera: contributed to conceive and designed the experiments and revised the paper.

Conflicts of Interest: The authors declare no conflict of interest. The founding sponsors had no role in the design of the study; in the collection, analyses, or interpretation of data; in the writing of the manuscript, and in the decision to publish the result.

\section{References}

1. Boutrif, E.; Canet, C. Mycotoxin prevention and control: FAO programmes. Rev. Méd. Vét. 1998, 149, 681-694.

2. Wild, C.P.; Gong, Y.Y. Mycotoxins and human disease: A largely ignored global health issue. Carcinogenesis 2010, 31, 71-82. [CrossRef] [PubMed]

3. Pitt, J.I.; Wild, C.P.; Baan, R.A.; Gelderblom, W.A.; Miller, D.J.; Riley, R.T.; Felicia, W.U. Improving Public Health through Mycotoxin Control; IARC Scientific Publications No. 157; World Health Organization: Lion, France, 2012.

4. Khlangwiset, P.; Shephard, G.S.; Wu, F. Aflatoxins and growth impairment: A review. Crit. Rev. Toxicol. 2011, 41, 740-755. [CrossRef] [PubMed]

5. Monographs on the Evaluation of Carcinogenic Risks to Humans. Some Naturally Occurring Substances: Food Items and Constituents, Heterocyclic Aromatic Amines and Mycotoxins. IARC; World Health Organization and International Agency for Research on Cancer: Lyon, France, 1993; Volume 56.

6. Smith, L.E.; Stoltzfus, R.J.; Prendergast, A. Food Chain Mycotoxin Exposure, Gut Health, and Impaired Growth: A Conceptual Framework. Adv. Nutr. 2012, 3, 526-531. [CrossRef] [PubMed] 
7. Shephard, G.S.; Burger, H.M.; Gambacorta, L.; Gong, Y.Y.; Krska, R.; Rheeder, J.P.; Solfrizzo, M.; Srey, C.; Sulyok, M.; Visconti, A.; et al. Multiple mycotoxin exposure determined by urinary biomarkers in rural subsistence farmers in the former Transkei, South Africa. Food Chem. Toxicol. 2013, 62, 217-225. [CrossRef] [PubMed]

8. Turner, P.C.; Flannery, B.; Isitt, C.; Ali, M.; Pestka, J. The role of biomarkers in evaluating human health concerns from fungal contaminants in food. Nutr. Res. Rev. 2012, 25, 162-179. [CrossRef] [PubMed]

9. Wild, C.P.; Turner, P.C. The toxicology of aflatoxins as a basis for public health decisions. Mutagenesis 2002, 17, 471-481. [CrossRef] [PubMed]

10. Kensler, T.W.; Roebuck, B.D.; Wogan, G.N.; Groopman, J.D. Aflatoxin: A 50 year odyssey of mechanistic and translational toxicology. Toxicol. Sci. 2011, 120, S28-S48. [CrossRef] [PubMed]

11. Groopman, J.D.; Jackson, P.E.; Turner, P.; Wild, C.P.; Kensler, T.W. Validation of Exposure and Risk Biomarkers: Aflatoxin As a Case Study. In Biomarkers of Environmentally Associated Disease Technologies, Concepts, and Perspectives; Wilson, S.H., William, A.S., Eds.; CRC Press: Boca Raton, FL, USA, 2002.

12. Levy, S.E.; Mandell, D.S.; Schultz, R.T. Autism. Lancet 2009, 374, 1627-1638. [CrossRef]

13. Goldberg, W.A.; Osann, K.; Filipek, P.A.; Laulhere, T.; Jarvis, K.; Modahl, C.; Flodman, P.; Spence, M.A. Language and other regression: Assessment and timing. J. Autism Dev. Disord. 2003, 33, 607-616. [CrossRef] [PubMed]

14. Lai, M.C.; Lombardo, M.V.; Auyeung, B.; Chakrabarti, B.; Baron-Cohen, S. Sex/gender differences and autism: setting the scene for future research. J. Am. Acad. Child Adolesc. Psychiatry 2015, 54, 11-24. [CrossRef] [PubMed]

15. Elsabbagh, M.; Divan, G.; Koh, Y.J.; Kim, Y.S.; Kauchali, S.; Marcín, C.; Montiel-Nava, C.; Patel, V.; Paula, C.S.; Wang, C.; et al. Global prevalence of autism and other pervasive developmental disorders. Autism Res. 2012, 5, 160-179. [CrossRef] [PubMed]

16. Liu, L.; Zhang, D.; Rodzinka-Pasko, J.K.; Li, Y.M. Environmental risk factors for autism spectrum disorders. Nervenartz 2016, 87, 55-61. [CrossRef] [PubMed]

17. Weber, T.K.; Polanco, I. Gastrointestinal microbiota and some children diseases: A review. Gastroenterol. Res. Pract. 2012, 676585. [CrossRef] [PubMed]

18. Sampson, T.R.; Mazmanian, S.K. Control of brain development, function, and behavior by the microbiome. Cell Host Microbe 2015, 17, 565-576. [CrossRef] [PubMed]

19. Strati, F.; Cavalieri, D.; Albanese, D.; De Felice, C.; Donati, C.; Hayek, J.; Jousson, O.; Leoncini, S.; Renzi, D.; Calabrò, A.; et al. New evidences on the altered gut microbiota in autism spectrum disorders. Microbiome 2017, 5, 24. [CrossRef] [PubMed]

20. Adams, J.B.; Johansen, L.J.; Powell, L.D.; Quig, D.; Rubin, R.A. Gastrointestinal flora and gastrointestinal status in children with autism-comparisons to typical children and correlation with autism severity. BMC Gastroenterol. 2011, 11. [CrossRef] [PubMed]

21. Pennesi, C.M.; Klein, L.C. Effectiveness of the gluten-free, casein-free diet for children diagnosed with autism spectrum disorder: Based on parental report. Nutr. Neurosci. 2012, 15, 85-91. [CrossRef] [PubMed]

22. EURACHEM, The Fitness for Purpose of Analytical Methods EURACHEM Guide; LGC: Teddington, UK, 1998.

23. Sulyok, M.; Berthiller, F.; Krska, R.; Schuhmacher, R. Development and validation of a liquid chromatography/tandem mass spectrometric method for the determination of 39 mycotoxins in wheat and maize. Rapid Commun. Mass Spectrom. 2006, 20, 2649-2659. [CrossRef] [PubMed]

24. Zimmerli, B.; Dick, R. Determination of ochratoxin-a at the ppt level in human blood, serum, milk and some foodstuffs by high-performance liquid-chromatography with enhanced fluorescence detection and immunoaffinity column cleanup-Methodology and swiss data. J. Chromat. B 1995, 666, 85-99. [CrossRef]

25. Heyndrickx, E.; Sioen, I.; Huybrechts, B.; Callebaut, A.; De Henauw, S.; De Saeger, S. Human biomonitoring of multiple mycotoxins in the Belgian population: Results of the BIOMYCO study. Environ. Int. 2015, 84, 82-89. [CrossRef] [PubMed]

26. Solfrizzo, M.; Gambacorta, L.; Visconti, A. Assessment of Multi-Mycotoxin Exposure in Southern Italy by Urinary Multi-Biomarker Determination. Toxins 2014, 6, 523-538. [CrossRef] [PubMed]

27. De Santis, B.; Brera, C.; Mezzelani, A.; Soricelli, S.; Ciceri, F.; Moretti, G.; Debegnach, F.; Bonaglia, M.C.; Villa, L.; Molteni, M.; et al. Role of Mycotoxins in the Pathobiology of Autism: A First Evidence. Nutr. Neurosci. 2017, under review. 
28. Brera, C.; De Santis, B.; Debegnach, F.; Miano, B.; Moretti, G.; Lanzone, A.; Del Sordo, G.; Buonsenso, D.; Chiaretti, A.; Hardie, L.; et al. External Scientific Report. In Experimental Study of Deoxynivalenol Biomarkers in Urine; EFSA (European Food Safety Authority): Parma, Italy, 2015; Volume 12, Available online: http:/ / onlinelibrary.wiley.com/doi/10.2903/sp.efsa.2015.EN-818/pdf (accessed on 9 June 2015).

29. International Agency for Research on Cancer (IARC). Monographs on the Evaluation of Carcinogenic Risks to Humans; IARC Press: Lyon, France, 2002; Volume 82. Available online: http:/ / monographs.iarc.fr/ENG/ Monographs/vol82/mono82.pdf (accessed on 9 June 2015).

30. Duringer, J.; Fombonne, E.; Craig, M. No Association between Mycotoxin Exposure and Autism: A Pilot Case-Control Study in School-Aged Children. Toxins 2016, 8, 224. [CrossRef] [PubMed]

31. Mayer, E.A.; Padua, D.; Tillisch, K. Altered brain-gut axis in autism: Comorbidity or causative mechanisms? Bioessays 2014, 36, 933-939. [CrossRef] [PubMed]

32. Buie, T.; Campbell, D.B.; Fuchs, G.J.; Furuta, G.T.; Levy, J.; Van de Water, J.; Whitaker, A.H.; Atkins, D.; Bauman, M.L.; Beaudet, A.L.; et al. Evaluation, Diagnosis, and Treatment of Gastrointestinal Disorders in Individuals With ASDs: A Consensus Report. Pediatrics 2010, 125, S1. [CrossRef] [PubMed]

33. Fulceri, F.; Morelli, M.; Santucci, E.; Cena, H.; Del Bianco, T.; Narzisi, A.; Calderoni, S.; Muratori, F. Gastrointestinal symptoms and behavioral problems in preschoolers with Autism Spectrum Disorder. Digest. Liver Dis. 2016, 48, 248-254. [CrossRef] [PubMed]

34. Matelski, L.; Van de Water, J. Risk factors in autism: Thinking outside the brain. J. Autoimmun. 2015, 67, 1-7. [CrossRef] [PubMed]

35. Napolioni, V.; Ober-Reynolds, B.; Szelinger, S.; Corneveaux, J.J.; Pawlowski, T.; Ober-Reynolds, S.; Kirwan, J.; Persico, A.M.; Melmed, R.D.; Craig, D.W.; et al. Plasma cytokine profiling in sibling pairs discordant for autism spectrum disorder. J. Neuroinflamm. 2013, 14, 38. [CrossRef] [PubMed]

36. Pecorelli, A.; Cervellati, F.; Belmonte, G.; Montagner, G.; Waldon, P.; Hayek, J.; Gambari, R.; Valacchi, G. Cytokines profile and peripheral blood mononuclear cells morphology in Rett and autistic patients. Cytokine 2016, 77, 180-188. [CrossRef] [PubMed]

37. Guo, S.; Nighot, M.; Al-Sadi, R.; Alhmoud, T.; Nighot, P.; Ma, T.Y. Lipopolysaccharide Regulation of Intestinal Tight Junction Permeability Is Mediated by TLR4 Signal Transduction Pathway Activation of FAK and MyD88. J. Immunol. 2015, 195, 4999-5010. [CrossRef] [PubMed]

38. Dal Peraro, M.; van der Goot, F.G. Pore-forming toxins: ancient, but never really out of fashion. Nat. Rev. Microbiol. 2016, 14, 77-92. [CrossRef] [PubMed]

39. Yamaguchi, N.; Sugita, R.; Miki, A.; Takemura, N.; Kawabata, J.; Watanabe, J.; Sonoyama, K. Gastrointestinal Candida colonisation promotes sensitisation against food antigens by affecting the mucosal barrier in mice. Gut 2006, 55, 954-960. [CrossRef] [PubMed]

40. Onore, C.E.; Nordahl, C.W.; Young, G.S.; Van de Water, J.A.; Rogers, S.J.; Ashwood, P. Levels of soluble platelet endothelial cell adhesion molecule-1 and P-selectin are decreased in children with autism spectrum disorder. Biol. Psychiatry 2012, 72, 1020-1025. [CrossRef] [PubMed]

41. Atkinson, W.; Sheldon, T.A.; Shaath, N.; Whorwell, P.J. Food elimination based on IgG antibodies in irritable bowel syndrome: A randomised controlled trial. Gut 2004, 53, 1459-1464. [CrossRef] [PubMed]

42. Lord, C.; Rutter, M.; DiLavore, P.C.; Risi, S.; Luyster, R.J.; Gotham, K.; Bishop, S.L.; Guthrie, W. ADOS-2-Autism Diagnostic Observation Schedule, 2nd ed.; Lord, C., Rutter, M., Eds.; Western Psychological Services: Torrance, CA, USA, 2012.

43. Lord, C.; Rutter, M.; Le Couteur, A. Autism diagnostic interview-revised: A revised version of a diagnostic interview for caregivers of individuals with possible pervasive developmental disorders. J. Autism Dev. Disord. 1994, 24, 659-685. [CrossRef] [PubMed]

44. Sparrow, S.S.; Balla, D.A.; Cichetti, D.V.; Reynolds, C.R. Vineland Adaptive-Behavior Scales. J. Couns. Dev. 1986, 65, 112-113.

45. Lam, K.S.; Aman, M.G. The Repetitive Behavior Scale-Revised: Independent validation in individuals with autism spectrum disorders. J. Autism Dev. Disord. 2007, 37, 855-866. [CrossRef] [PubMed]

46. Griffiths, R. Mental Development Scales from Birth to 2 Years; Manual; Association for Research in Infant and Child Development: Henley, UK, 1996.

47. Wechsler, D. Wechsler Preschool and Primary Scale of Intelligence-Revised 517 (WPPSI-R); Psychological Corporation: San Antonio, TX, USA, 1989. 
48. Wechsler, D. WISC-III: Wechsler Intelligence Scale for Children, 3rd ed.; The Psychological Corporation: New York, NY, USA, 1991.

49. Achenbach, T.M.; Rescorla, L.A. Manual for the ASEBA Preschool Forms and Profiles; University of Vermont Department of Psychiatry: Burlington, VT, USA, 2000.

50. Achenbach, T.M.; Rescorla, L.A. Manual for the ASEBA School-Age Forms and Profiles; University of Vermont, Research Center for Children, Youth and Families: Burlington, VT, USA, 2001.

51. D'Arcangelo, D.; Facchiano, F.; Nassa, G.; Stancato, A.; Antonini, A.; Rossi, S.; Senatore, C.; Cordella, M.; Tabolacci, C.; Salvati, A.; et al. PDGFR-alpha inhibits melanoma growth via CXCL10/IP-10: A multi-omics approach. Oncotarget 2016, 22, 77257-77275. [CrossRef] [PubMed]

52. Wilcoxon, F. Individual comparisons by ranking methods. Biometrics 1945, 1, 80-83. [CrossRef]

53. Mann, H.B.; Whitney, D.R. On a test whether one of two random variables is stochastically larger than the other. Ann. Math. Stat. 1947, 18, 50-60. [CrossRef]

54. Kruskal, W.H.; Wallis, W.A. Use of ranks in one-criterion variance analysis. J. Am. Stat. Assoc. 1952, 47, 583-621. [CrossRef]

55. Maranghi, F.; Baldi, F.; Mantovani, A. Sicurezza Alimentare e Salute Dell'Infanzia; Rapporti ISTISAN 05/35; Istituto Superiore di Sanità: Rome, Italy, 2005; Volume 3, p. 139.

56. Ubagai, T.; Tansho, S.; Ito, T.; Ono, Y. Influences of aflatoxin B1 on reactive oxygen species generation and chemotaxis of human polymorphonuclear leukocytes. Toxicol. In Vitro 2008, 22, 1115-1120. [CrossRef] [PubMed]

57. Richetti, A.; Cavallaro, A.; Ainis, T.; Fimiani, V. Effect of mycotoxins on some activities of isolated human neutrophils. Immunopharmacol. Immunotoxicol. 2005, 27, 433-446. [CrossRef] [PubMed]

58. Gauthier, T.; Waché, Y.; Laffitte, J.; Taranu, I.; Saeedikouzehkonani, N.; Mori, Y.; Oswald, I.P. Deoxynivalenol impairs the immune functions of neutrophils. Mol. Nutr. Food Res. 2013, 57, 1026-1036. [CrossRef] [PubMed]

59. Melchionna, R.; Romani, M.; Ambrosino, V.; D’Arcangelo, D.; Cencioni, C.; Porcelli, D.; Toietta, G.; Truffa, S.; Gaetano, C.; Mangoni, A.; et al. Role of HIF-1alpha in proton-mediated CXCR4 down-regulation in endothelial cells. Cardiovasc. Res. 2010, 86, 293-301. [CrossRef] [PubMed]

60. Yap, C.C.; Winckler, B. Acid indigestion in the endosome: Linking signaling dysregulation to neurodevelopmental disorders. Neuron 2013, 80, 4-6. [CrossRef] [PubMed]

61. Cash-Padgett, T.; Sawa, A.; Jaaro-Peled, H. Increased stereotypy in conditional Cxcr4 knockout mice. Neurosci. Res. 2016, 105, 75-79. [CrossRef] [PubMed] 